\title{
Bioinspired Propulsion Mechanism in Fluid Using Fin with Dynamic Variable-Effective-Length Spring*
}

\author{
Shunichi KOBAYASHI ${ }^{* *}$ Masataka NAKABAYASHI ${ }^{* *}$ and Hirohisa MORIKAWA ${ }^{* *}$ \\ ${ }^{* *}$ Department of Functional Machinery and Mechanics, \\ Faculty of Textile Science and Technology, Shinshu University \\ 3-15-1 Tokida, Ueda, Nagano, 386-8567 Japan \\ E-mail: shukoba@shinshu-u.ac.jp
}

\begin{abstract}
Since the propulsion mechanism using elastic fins, such as the caudal fin and pectoral fin of fish, is effective in fluid, many studies on bioinspired elastic fins for propulsion in water and on the development of fish-type robots with elastic fins have been carried out. The optimum elasticity of the fin is not constant and varies according to the movement task and environment, such as swimming speed and oscillating frequency. However, it is very difficult to exchange fins of different stiffnesses while moving. Thus, we aimed to develop a variable-stiffness fin of which stiffness can be changed dynamically. As the one such variable-stiffness fin, we have developed a fin with a variable-effective-length spring. The effective length of a plate spring is changed by adjusting the length of the rigid plate that supports the plate spring. Apparent stiffness is changed by varying the effective length. In this paper, we have described the structure of the propulsion mechanism in fluid using a fin with a variable-effective-length spring, and the thrust force characteristics in water. Furthermore, we have discussed the optimum effective length for providing the maximum thrust force and the effect of the dynamic change of effective length on thrust force.
\end{abstract}

Key words: Propulsion Mechanism, Fin, Variable-Effective-Length Spring

\section{Introduction}

The conventional screw propeller is the general propulsion system for ships or underwater vehicles. As an alternative propulsion mechanism for higher propulsion efficiency and better safety than the screw propeller, propulsion by oscillating an elastic fin resembling a caudal fin or pectoral fin of fish has been proposed, and a basic study on the bioinspired oscillating fin and its development as a propulsion method for ships or underwater vehicles/robots have been carried out ${ }^{(1)-(3)}$. The optimum elasticity of a fin is not constant and changes according to the movement task and environment, such as swimming speed and oscillating frequency ${ }^{(2),(3)}$. However, it is very difficult to exchange fins of different bending stiffnesses while moving. On the other hand, fish are able to move their fins dynamically and can change their resistance to external force. Fish may be able to change the apparent stiffness of their fins by muscle contraction, though further investigations of fin stiffness for fish are needed.

We aimed to develop a bioinspired propulsion mechanism using a variable-bending-stiffness fin of which stiffness can be changed dynamically. There are some mechanisms of dynamic variable stiffness ${ }^{(4),(5)}$. However, there has been no study of a

${ }^{*}$ Received 21 May, 2006 (No. 06-0035) [DOI: 10.1299/jbse.1.280] 
dynamic variable-stiffness mechanism for propulsion in fluid. As the first task of this study, we studied a variable-bending-stiffness fin modeled on ciliary movement, using electromagnets to control friction resistance between two flexible plates ${ }^{(6)}$. However, this model was small and it was difficult to generate sufficient thrust force for the oscillating fin. For the development of a propulsion mechanism using an oscillating fin, we have made a variable-bending-stiffness fin with a variable-effective-length spring. The apparent bending stiffness of the fin can be changed dynamically. In this paper, we describe the structure of the propulsion mechanism in fluid using a fin with a variable-effective-length spring, and the characteristics of thrust force with changing effective length of the spring. Furthermore, we discuss the effect of a dynamic change of the effective length of a spring from the viewpoint of generating greater thrust force.

\section{Structure of Propulsion Mechanism in Fluid}

Figure 1 shows the principle of the variable-effective-length spring. Effective length of the spring $H$ is changed by varying the length of rigid plates supporting the plate spring made of polyethylene terephthalate, (PET). The relationship between the displacement of the plate spring and effective length $H$ (constant lateral load: 1.96N) is shown in Fig. 2. Apparent stiffness changes with $H$. Figure 3 shows the fin with a variable-effective-length spring. The fin system consists of an aluminum box (length: $130 \mathrm{~mm}$, height: $60 \mathrm{~mm}$, width: $25 \mathrm{~mm}$ ), a fin made of a chloride plate (length: $120 \mathrm{~mm}$, height: $60 \mathrm{~mm}$, thickness: $3 \mathrm{~mm}$ ), and a variable-effective-length spring (length $L: 20 \mathrm{~mm}$, height: $43 \mathrm{~mm}$, thickness: $0.5 \mathrm{~mm}$ ). A DC motor, waterproof bearing, slipping screw, resin nut and rigid plate are housed in the aluminum box. A transparent chloride plate cover is attached on the open side of the aluminum box to seal it. Figure 4 shows the experimental system. The fin is moved in a stationary water tank (length: $2020 \mathrm{~mm}$, width: $730 \mathrm{~mm}$, depth: $250 \mathrm{~mm}$ ). The fin base is positioned at the middle of the length and width of the water tank, and the upper surface of the fin is $90 \mathrm{~mm}$ below the water level. The propulsion mechanism is supported by a linear guide and is able to move. For the measurement of thrust force while the mechanism is stationary on the linear guide, the mechanism is connected to a load cell (NEC San-ei Instruments, T1). The pitching movement of the fin is driven by the geared DC servomotor (Maxon Motor, 11875, HEDL5544, 114492). We can change the maximum pitching angle $\theta_{\max }$ and movement cycle $T$. The signal from the load cell is amplified by a strain amplifier (NEC San-ei Instruments, As1603) and recorded on a personal computer in synchrony with the control of the DC servomotor, using LabView7 software (National Instruments).

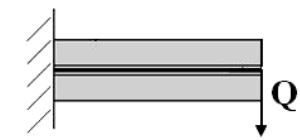

(a) Apparent stiffness : Maximum $(\mathrm{H}=\mathbf{0})$

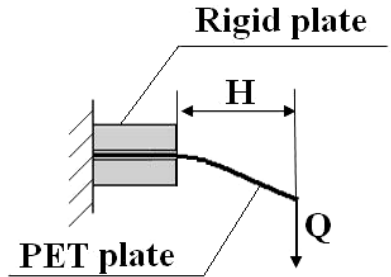

(b) Apparent stiffness : Middle $(\mathbf{0}<\mathbf{H}<\mathbf{L})$

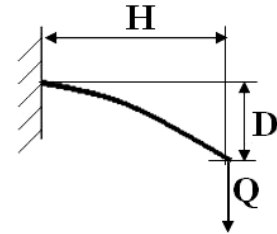

(c) Apparent stiffness : Minimum ( $\mathbf{H}=\mathbf{L})$

$Q$ : Load $H$ : Effective-length $L$ : Maximum effective-length D : Displacement

Fig. 1 Principle of effective length spring 


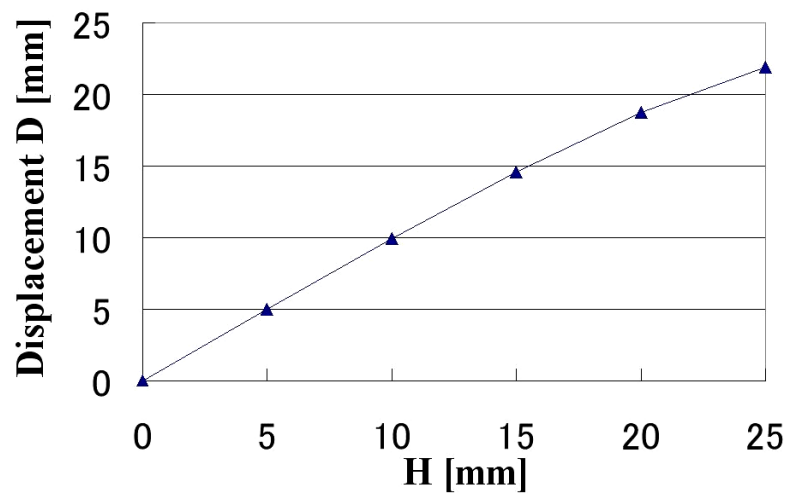

Fig. 2 Relationship between displacement of plate spring $D$ and effective length of spring $H$. Total length of spring $L=25 \mathrm{~mm}$. Thickness of spring $t=0.5 \mathrm{~mm}$. Constant lateral load $Q=1.96 \mathrm{~N}$.

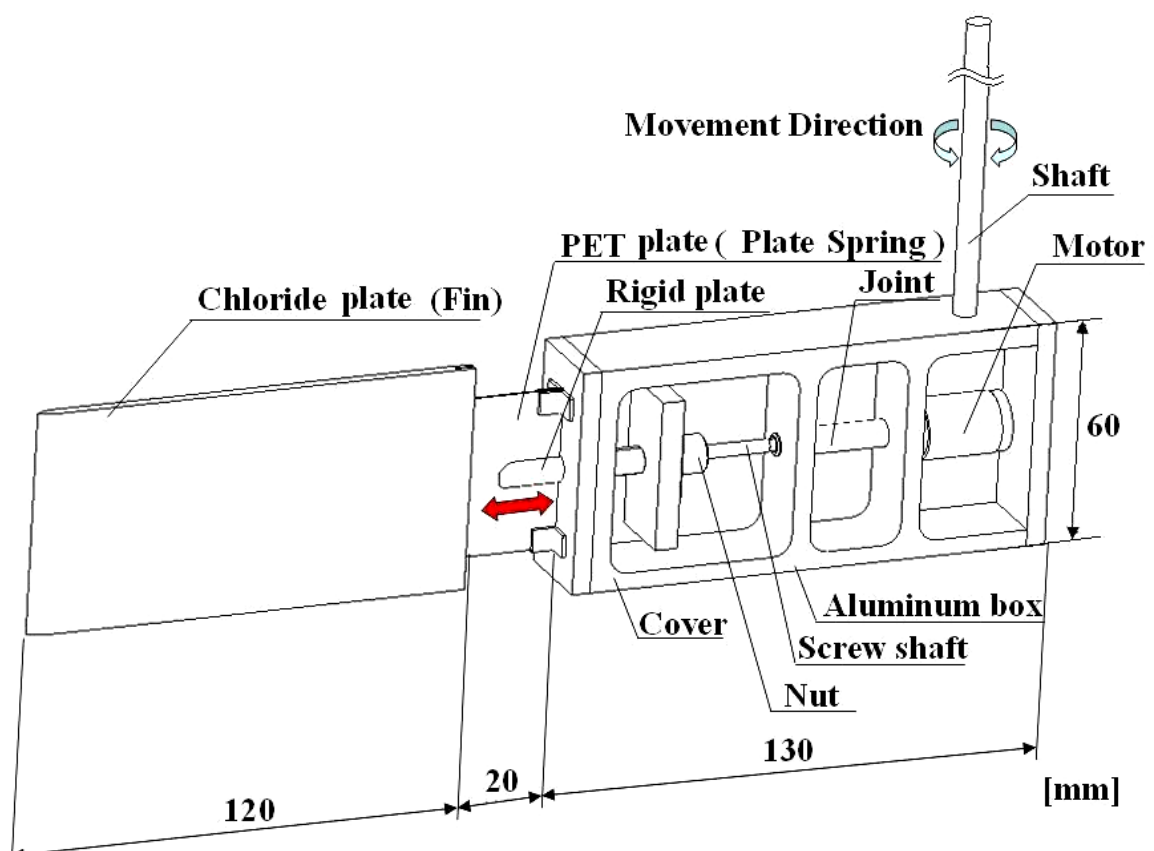

Fig. 3 Fin with variable-effective-length spring

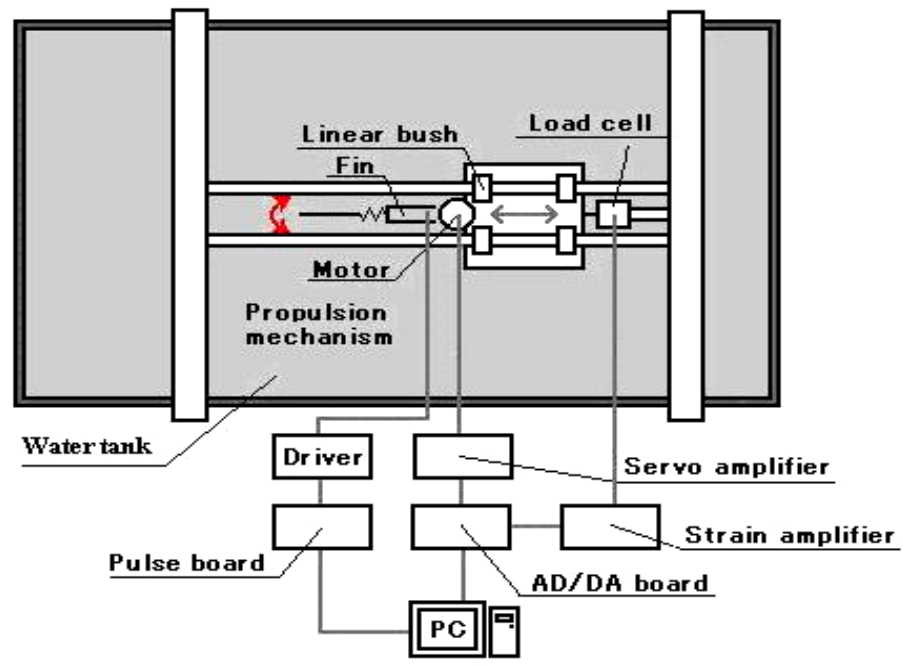

Fig. 4 Experimental system 


\section{Results and Discussion}

\subsection{Behavior of Oscillating Fin}

Figure 5 shows the change in fin shape and bending angle $\alpha$ in one movement cycle. The effective spring length $H$ is fixed during motion (Static condition of $H$ ). Bending angle $\alpha$ is changed by the fluid resistance during motion. $\alpha$ increases with increasing $H$, that is, with a decrease in apparent stiffness. When $H=0$ (no effective length of spring), $\alpha$ is small but not 0 . This small bending is due to small gap between the top of the rigid plate and the chloride plate (fin) when $H=0$.

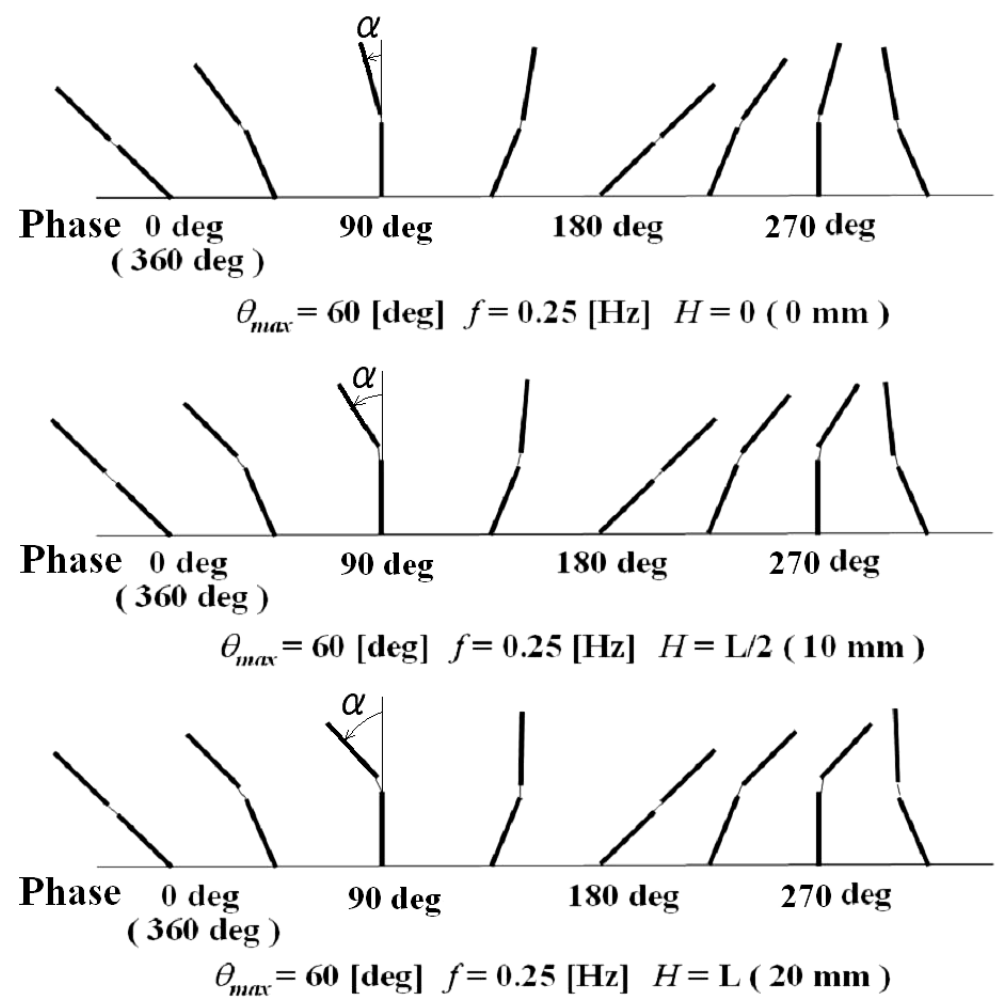

(a) Fin shape $(T=4 \mathrm{~s})$.

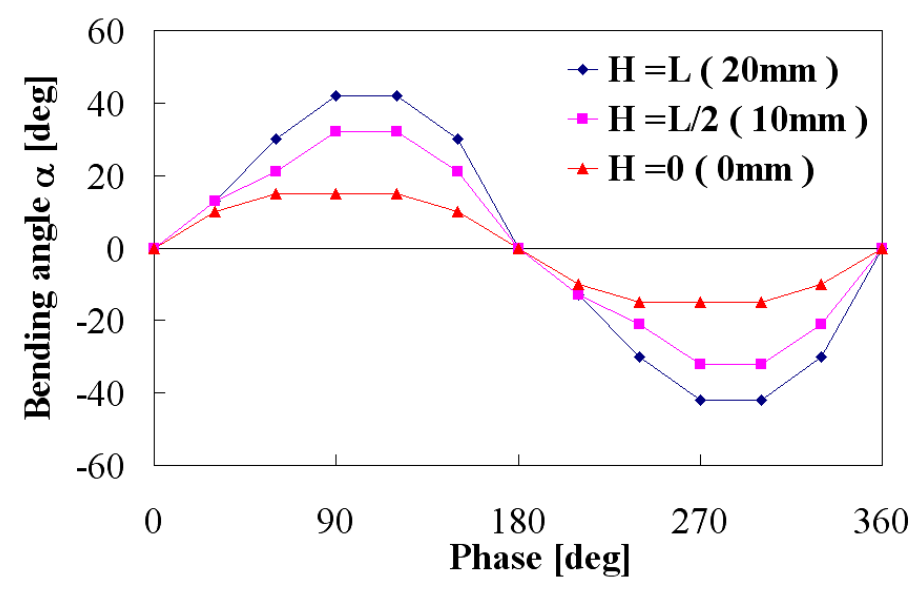

(b) Bending angle $\left(T=4 \mathrm{~s}, \theta_{\max }=45 \mathrm{deg}\right)$.

Fig. 5 Change of fin shape and bending angle $\alpha$ in one movement cycle. 


\subsection{Relationship between Effective Spring Length and Thrust Force}

Figure 6 shows the variation of thrust force in one movement cycle. The effective length of spring $H$ was fixed during motion (static condition of $H$ ). With $H=0$ as the maximum apparent stiffness condition, the difference between maximum thrust force $F_{\max }$ and minimum thrust force $F_{\min }$, and the magnitude of the negative thrust force are greater than those when $H=L$ and $H=L / 2$. Average thrust forces when $H=L$ and $H=L / 2$ are greater than that when $H=0$. These results indicate that an elastic fin is favorable realizing the effective thrust force. The phases of $F_{\max }$ and $F_{\min }$ when $H=L$ and $H=L / 2$ are delayed compared with those when $H=0$. This is because the phase of the fin shape to generate a great magnitude of thrust force is delayed by the elastic bending.

Figure 7 shows the relationship between average thrust force $F_{\text {avg }}$ and effective length $H$. In this figure, average angular velocity $\omega_{\text {avg }}=2 \theta_{\max } / T$ is indicated, and "Max." marks the maximum $F_{\text {avg }}$ under each condition. If we define the optimum effective length $H_{\text {opt }}$ as the length required to generate maximum thrust force, $H_{o p t}$ generally increases with increasing movement cycle $T$. However, this tendency is rarely observed at smaller bending angles (maximum pitching angle $\theta_{\max }=30 \mathrm{deg}$ ). The reason for this may be that the angular velocity of the fin is lower for smaller $\theta_{\max }$, thus the effect of elastic bending is also weaker. Figure 8 shows the relationship between the standard deviation of thrust force in one movement cycle $F_{\text {std }}$ and the effective length of the spring $H$. It is apparent from the figure that $F_{\text {std }}$ decreases with increasing $H$, that is, thrust force stabilizes with a decrease in apparent bending stiffness.

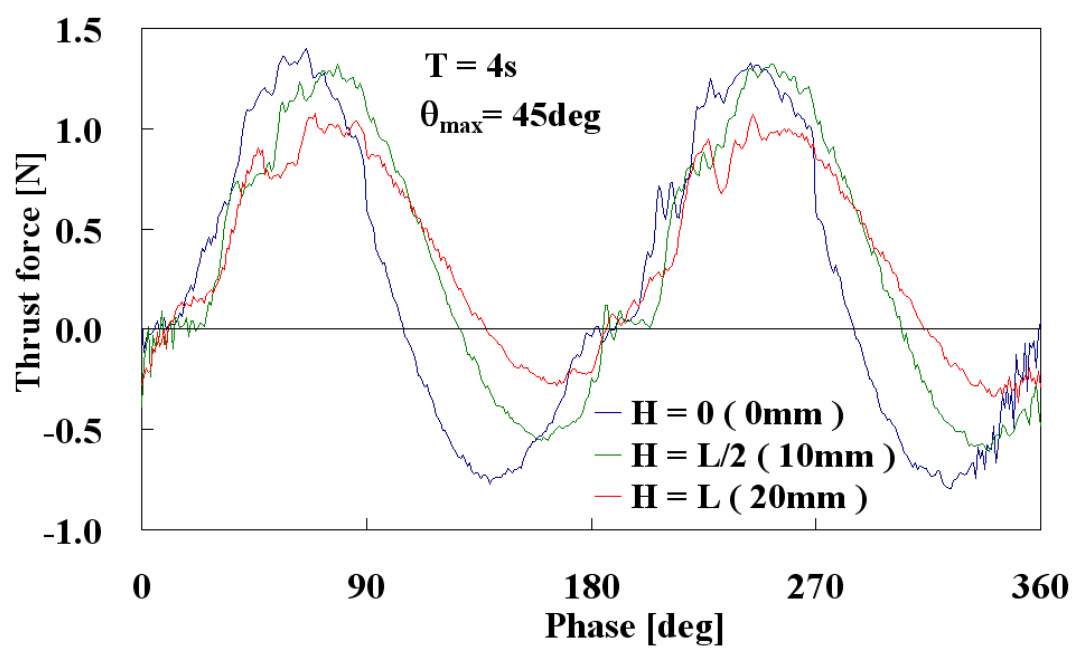

Fig. 6 Variation of thrust force in one movement cycle $\left(T=4 \mathrm{~s}, \theta_{\max }=45 \mathrm{deg}\right)$. 


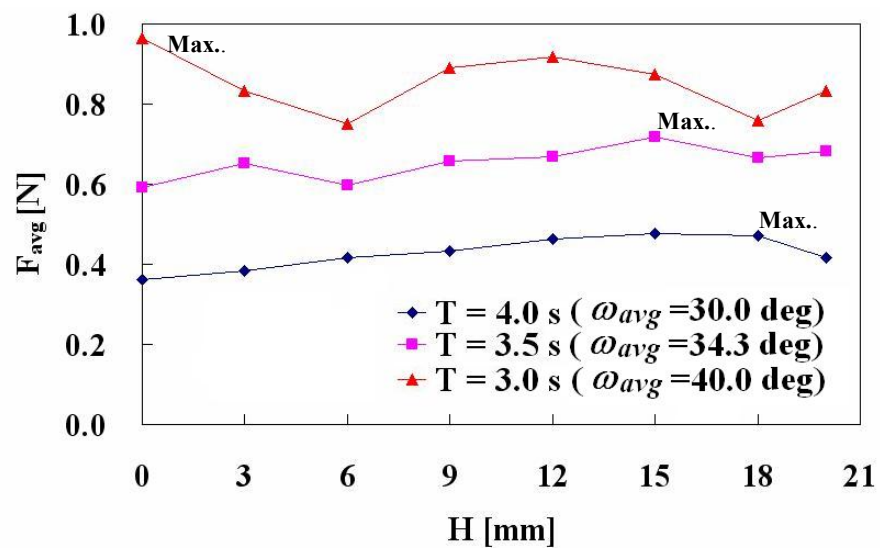

(a) $\theta_{\max }=60 \mathrm{deg}$

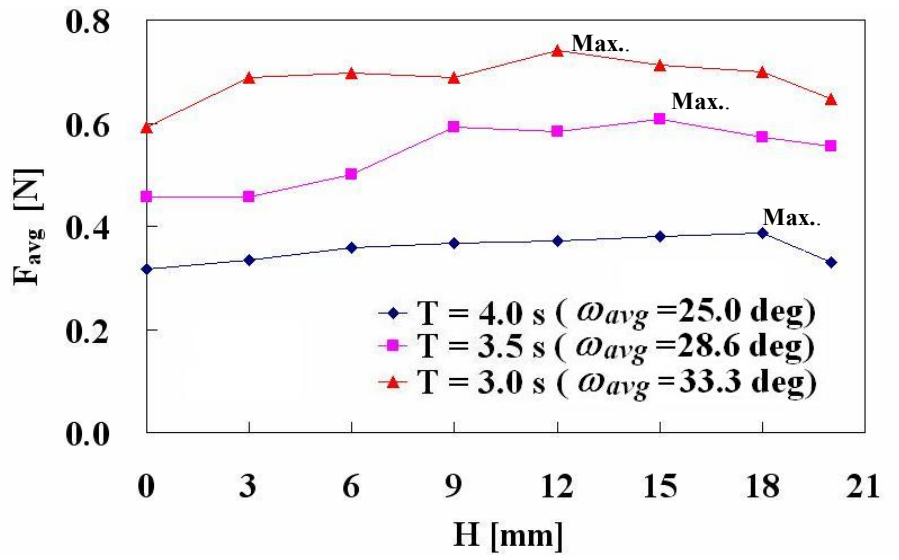

(b) $\theta_{\max }=50 \mathrm{deg}$

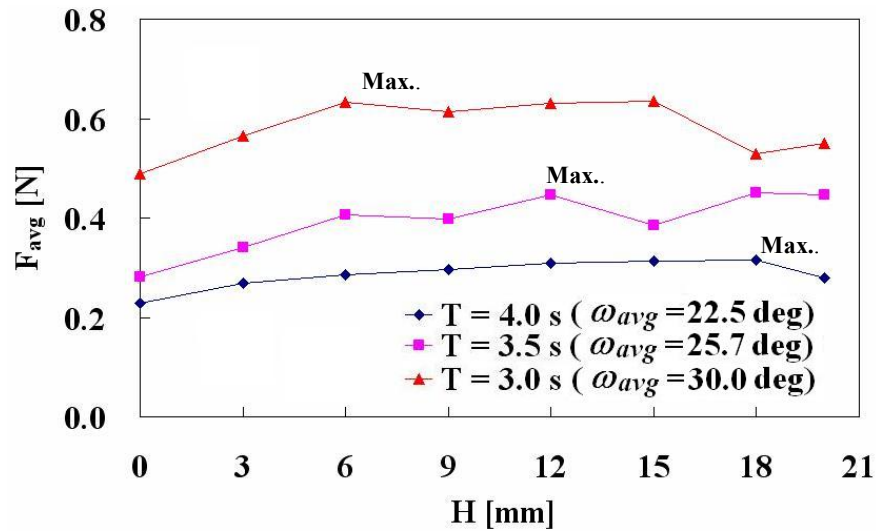

(c) $\theta_{\max }=45 \mathrm{deg}$

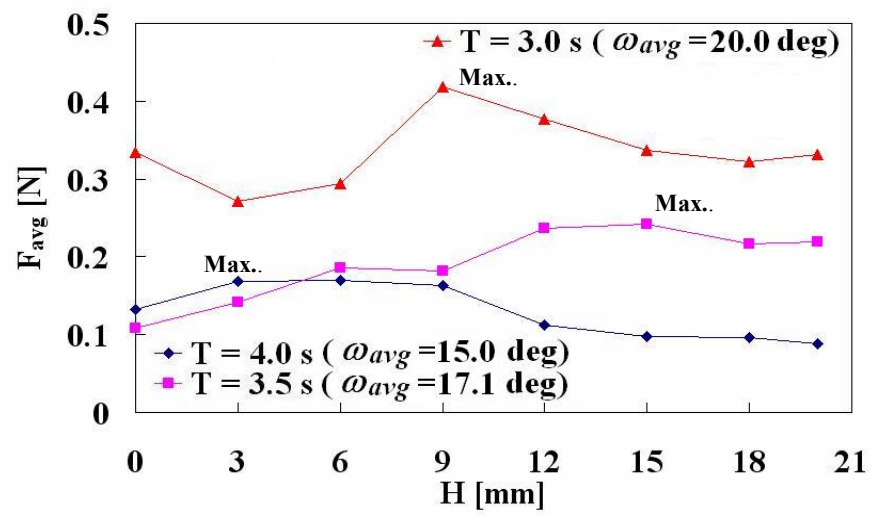

(d) $\theta_{\max }=30 \mathrm{deg}$

Fig. 7 Relationship between average thrust force $F_{a v g}$ and effective length $H$. 


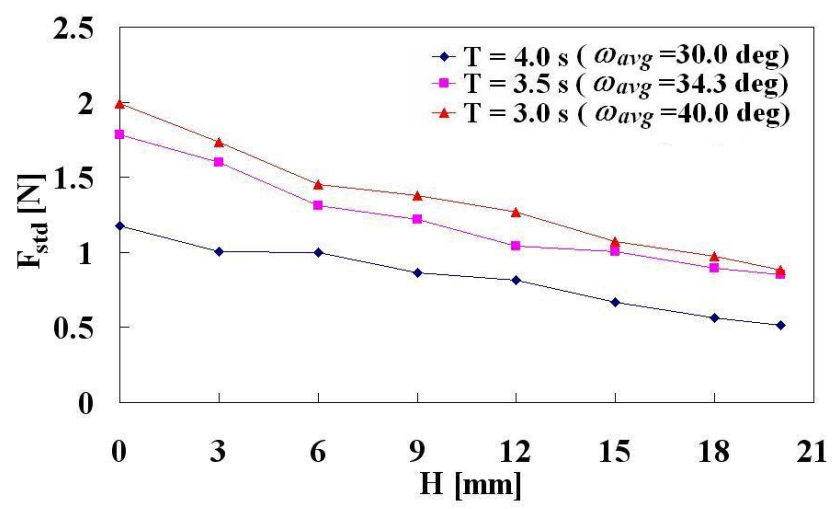

(a) $\theta_{\max }=60 \mathrm{deg}$

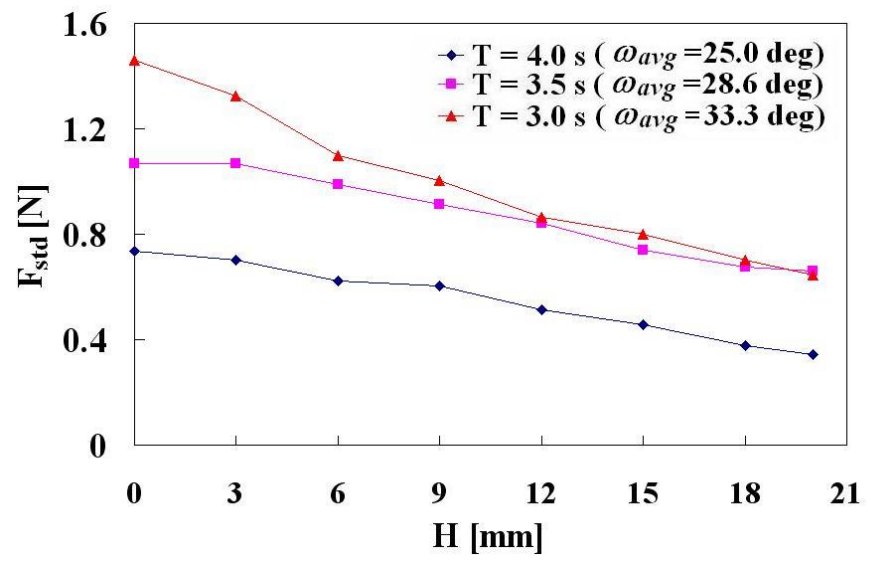

(b) $\theta_{\max }=50 \mathrm{deg}$

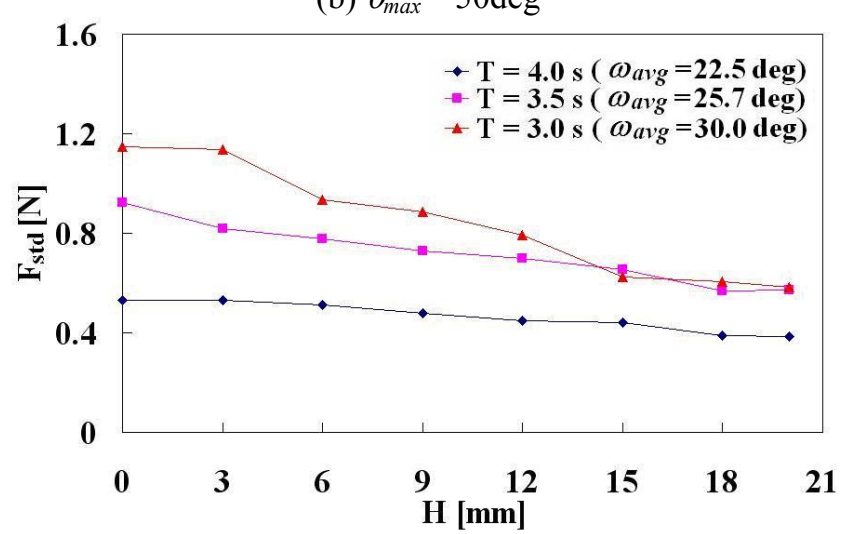

(c) $\theta_{\max }=45 \mathrm{deg}$

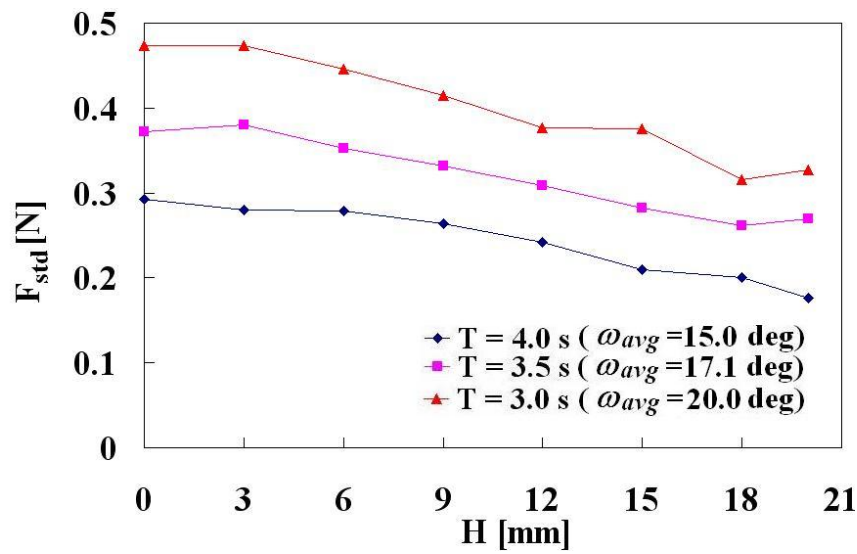

(d) $\theta_{\max }=30 \mathrm{deg}$

Fig. 8 Relationship between standard deviation of thrust force in one movement cycle $F_{\text {std }}$ and effective length of spring $H$. 


\subsection{Dynamic Change of Effective Length}

After the experiments under the static condition of effective length of the spring $H$, we controlled the dynamic change of effective length during motion. Figure 9 shows the change of $H$ and thrust force in one movement cycle. $H$ is controlled to obtain greater thrust force. For the phases of $0-90 \mathrm{deg}$ and $180-270 \mathrm{deg}, H$ was set at $0 \mathrm{~mm}$ because the thrust forces in these phases are greater when $H=0 \mathrm{~mm}$ (static condition of $H$, shown in Fig. 6). For the phases of 90-180deg and 270-360deg, $H$ was set at $20 \mathrm{~mm}$ because the thrust forces in these phases are greater when $H=20 \mathrm{~mm}$ (static condition of $H$, shown in Fig. 6). In Fig. 9 , thrust forces during the phases of $0-90 \mathrm{deg}$ and 180-270deg are the closer to those when $H=0 \mathrm{~mm}$ shown in Fig. 6, and thrust forces during the phases of 90-180deg and 270-360deg are closer to those when $H=20 \mathrm{~mm}$ shown in Fig. 6. Thus the average thrust force with the dynamic change of $H$ is greater than that under the static condition of $H$. Table 1 shows average thrust force $F_{\text {avg }}$ and standard deviation of thrust force $F_{\text {std }}$ for optimum effective length $H_{\text {opt }}$ (static condition of $H$ ) and dynamic change of $H$. Average thrust force $F_{\text {avg }}$ for the dynamic change of $H$ is greater than that under the static condition of $H$ when $\theta_{\max }$ is $45 \mathrm{deg}(\mathrm{T}=4 \mathrm{~s})$ and $60 \mathrm{deg}(\mathrm{T}=3 \mathrm{~s}, 4 \mathrm{~s})$. However, the standard deviation of thrust force is greater than that under the static condition of $H$, because we have not regarded the variance of thrust force. Optimum control of $H$ for achieving greater and stable thrust force is needed in the future.

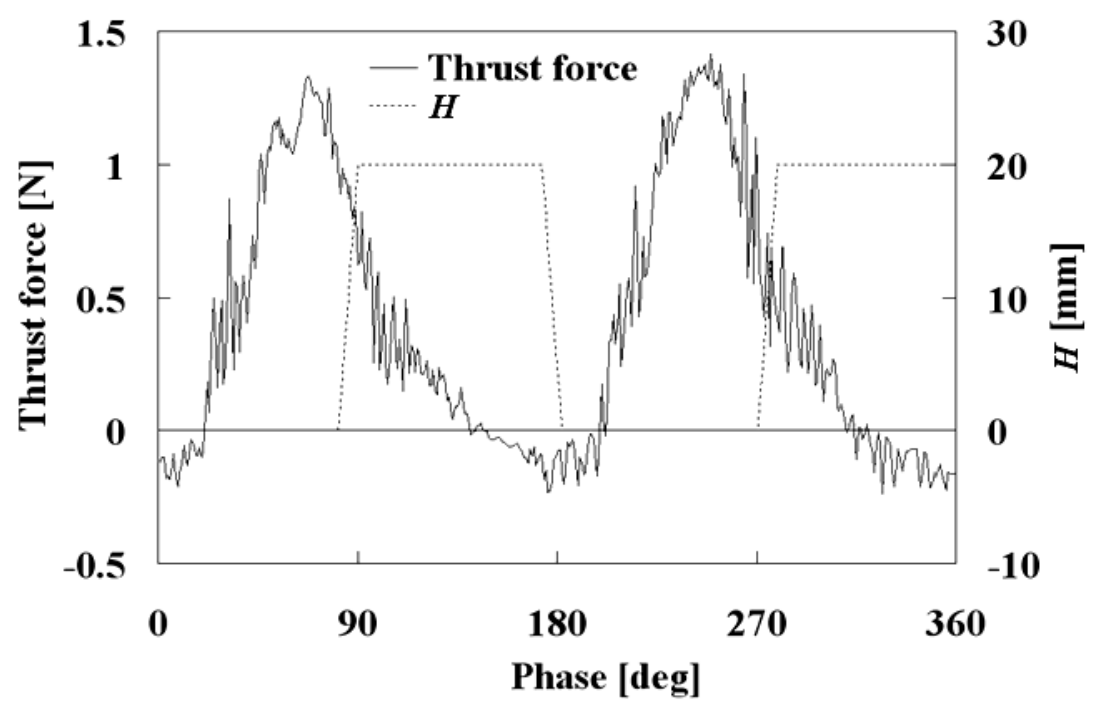

Fig. 9 Change of effective length of spring $H$ and variation of thrust force in one movement cycle ( $\left.T=4 \mathrm{~s}, \theta_{\max }=45 \mathrm{deg}\right)$. 
Table 1 Average thrust force $F_{\text {avg }}$ and standard deviation of thrust force $F_{\text {std }}$ with optimum effective length of spring $H_{\text {opt }}$ for obtaining the greatest thrust force (static condition of $H$ ) and dynamic change of effective length of spring.

\begin{tabular}{|c|c|c|c|c|c|}
\hline \multirow{2}{*}{$\mathrm{T}[\mathrm{s}]$} & & \multirow{2}{*}{ Condition of $H$} & \multicolumn{3}{|c|}{$\theta_{\max }[\mathrm{deg}]$} \\
\hline & & & 30 & 45 & 60 \\
\hline \multirow{4}{*}{3} & \multirow{2}{*}{$F_{\text {avg }}[\mathrm{N}]$} & Static* & 0.42 & 0.64 & 0.96 \\
\hline & & Dynamic*** & 0.42 & 0.64 & 1.53 \\
\hline & \multirow{2}{*}{$F_{\text {std }}[\mathrm{N}]$} & Static* & 0.41 & 0.94 & 1.27 \\
\hline & & Dynamic $* * *$ & 0.42 & 0.97 & 1.55 \\
\hline \multirow{4}{*}{4} & \multirow{2}{*}{$F_{\text {avg }}[\mathrm{N}]$} & Static** & 0.17 & 0.31 & 0.46 \\
\hline & & Dynamic*** & 0.17 & 0.39 & 0.58 \\
\hline & \multirow{2}{*}{$F_{\text {std }}[\mathrm{N}]$} & Static** & 0.26 & 0.48 & 0.57 \\
\hline & & Dynamic*** & 0.27 & 0.50 & 0.85 \\
\hline
\end{tabular}

\section{Conclusions}

This study was conducted to develop a propulsion mechanism in fluid, using a fin with a dynamic variable-effective-length spring. We discussed the influence of thrust force on the effective length of the spring under static and dynamic conditions. The following results were obtained.

(1) Bending angle $\alpha$ of the fin increases with an increase in effective length of the spring $H$.

(2) Under the static condition of effective length, optimum length $H_{\text {opt }}$ for obtaining greater thrust force generally increases with increasing movement cycle $T$.

(3) Average thrust force $F_{\text {avg }}$ with the dynamic change of $H$ is greater than that under the static condition of $H$ when $\theta_{\max }$ is $45 \mathrm{deg}(T=4 \mathrm{~s})$ and $60 \mathrm{deg}(T=3 \mathrm{~s}, 4 \mathrm{~s})$.

Since this is the first study on using a variable-effective-length spring for propulsion in fluid, further research is still needed. The issues to be tackled in the future include the following:

(1) Addition of heaving movement mechanism.

(2) Discussion of efficiency.

(3) Optimum control of the effective length for achieving greater and stable thrust force.

(4) Measurement in a water tunnel and flow visualization.

\section{Acknowledgements}

This work was supported by a research grant from the Fundamental Research Developing Association for Shipbuilding and Offshore, and by a Grant-in-Aids for Scientific Research (16560224-00) by the Japan Society for the Promotion of Science.

\section{References}

(1) Morikawa, H. and Isshiki, N., The Study on a Propulsion System by Fin Stroke, Bulletin of M.E.S.J., Vol. 8, No. 1, (1980).

(2) Nakajima, H., Tokuo, K., and Ono, K., Experimental Study of a Two-Joint Dolphinlike Propulsion Mechanism (2nd Report, Experiment of Self-Propelled Large Robot), Transactions of the Japan Society of Mechanical Engineers, Series C, Vol. 66, №.643, (2000), pp. $695-702$.

(3) Watanabe, M., Muramatsu, K., and Kobayashi, N., Propulsion Performance of an Aquatic 
Mobile Robot Using Traveling-Wave Motion of a Flexible Fin (Relationship between Propulsion Efficiency and Flow Pattern, Transactions of the Japan Society of Mechanical Engineers, Series C, Vol. 68, No. 665, (2002), pp. 188-196.

(4) Kawamura, S., Yamamoto, T., Ishida, D. Ogata, T., Nakayama, Y., Tabata, O., and Sugiyama, S., Development of Passive Elements with Variable Mechanical Impedance for Wearable Robots, Proceedings of 2002 IEEE International Conference on Robotics and Automation, (2002), pp.11-15.

(5) Umedachi, T., Kishi, H., Yamada, Y., and Ishiguro, A., Development of a Real-time Tunable Spring -Toward Independent Control of Position and Stiffness of Joints-, Proceedings of the 2005 JSME Conference on Robotics and Mechatronics, (CDROM), (2005), 1P2-S-005.

(6) Kobayashi, S., Mashima, T., and Morikawa, H., Thrust Force Characteristics of Propulsion Mechanism in Fluid Using Variable-Bending-Stiffness Fin Modeled on Ciliary Movement, JSME International Journal, Series C, Vol. 46, No. 4, (2003), pp. 1340 - 1345. 